\title{
Predictive Values of Serum Biochemical Markers and Apparent Diffusion Coefficient on Delayed Encephalopathy After Acute Carbon Monoxide Poisoning
}

\author{
Runming SUN ${ }^{1 *}$, Wenyuan $\mathrm{CAO}^{1 *}$, Zhangge $\mathrm{Jl}^{1 *}$, Wenwen BIAN ${ }^{1 *}$, LiXia WANG ${ }^{2 \#}$, Qian WANG ${ }^{1 \#}$, Zhaodong $\mathrm{LI}^{3}$ \\ 'Zibo Municipal Hospital, Department of Neurology, Zibo City, Shandong Province, China \\ ${ }^{2}$ Zibo Municipal Hospital, Department of Endocrine, Zibo City, Shandong Province, China \\ ${ }^{3}$ Zibo Municipal Hospital, Department of Radiology, Zibo City, Shandong Province, China \\ ${ }^{*}$ Runming Sun, Wenyuan Cao, Zhangge Ji and Wenwen Bian are co-first authors \\ "LiXia Wang and Qian Wang are co-second authors
}

Corresponding author: Zhaodong LI lizhaodongs002@163.com

\section{ABSTRACT}

AIM: To explore the predictive values of serum biochemical markers and apparent diffusion coefficient (ADC) on delayed encephalopathy after acute carbon monoxide poisoning (DEACMP).

MATERIAL and METHODS: Seventy-nine patients were divided into two groups based on the onset of DEACMP 60 days after with acute carbon monoxide poisoning. The baseline data of ACMP patients were collected. Serum levels of cardiac troponin I (cTNI), lactic acid (Lac), carboxyhemoglobin ( $\mathrm{COHb}$ ), creatine kinase $(\mathrm{CK})$, creatine kinase isoenzyme (CK-MB), lactate dehydrogenase $(\mathrm{LDH})$, C-reactive protein (CRP), and neuron-specific enolase (NSE) were measured within 24 hours of ACMP onset. The ADC of globus pallidum, centrum semiovale, and periventricular white matter were recorded by diffusion-weighted magnetic resonance imaging (DW-MRI) within 48 hours of admission. Logistic regression analysis was performed to determine the risk factors for DEACMP.

RESULTS: The incidence of coma and duration of poisoning in the DEACMP group were significantly higher than those in the non-DEACMP group. The levels of Lac, CK, CK-MB, LDH, CRP and NSE in the DEACMP group were higher than those in the nonDEACMP group. The ADC value of globus pallidus in the DEACMP group was significantly higher than that in non-DEACMP group. The duration of poisoning, coma degree, CK, LDH, NES, and CRP were closely associated with the occurrence of DEACMP.

CONCLUSION: The duration of poisoning, coma degree, and serum levels of CK, LDH, CRP, and NSE were independent risk factors for DEACMP.

KEYWORDS: Carbon monoxide poisoning, Coma, Creatine kinase, Neuron-specific enolase, Magnetic resonance imaging

ABBREVIATIONS: ADC: Apparent diffusion coefficient, cTNI: Cardiac troponin I, Lac: Lactic acid, COHb: Carboxyhemoglobin, CK: Creatine kinase, CK-MB: Creatine kinase isoenzyme, LDH: Lactate dehydrogenase, CRP: C-reactive protein, NSE: Neuron-specific enolase

Runming SUN (1) : 0000-0002-4795-945X Wenyuan CAO (1): :0000-0002-2349-6497

Zhangge $\mathrm{Jl}$ (1) : 0000-0002-3030-6532
Wenwen BIAN (10: : 0000-0003-1631-8163

LiXia WANG (10): 0000-0003-3275-3464

Qian WANG (1) : 0000-0001-8713-9918
Zhaodong LI (1) : 0000-0003-4103-0348 


\section{- INTRODUCTION}

$\Lambda$ cute carbon monoxide poisoning (ACMP) is the most common type of fatal air poisoning, causing dysfunction of the cardiovascular and central nervous system (26). Delayed encephalopathy after ACMP (DEACMP) is one of its most serious complications (11). DEACMP is characterized by a series of neuropsychiatric symptoms such as extrapyramidal symptoms, dementia, and mental disorders $(9,33)$. Patients with DEACMP have varying degrees of hypoxia and nerve cell damage (35). Sex, age, and degree of intoxication are significantly different among patients with DEACMP (47), thereby warranting efforts to explore new diagnostic methods for DEACMP.

Numerous studies have investigated the relationship between the values of serum biochemical factors and the incidence of late-onset disorder after ACMP. Wang et al. have reported that blood lactic acid (Lac) clearance rate in ACMP patients is negatively related to the incidence of DEACMP (39). Zhang et al. have found that the serum levels of creatine kinase isoenzyme (CK-MB) and lactate dehydrogenase (LDH) are markedly increased in patients with ACMP (46). Cha et al. have shown that serum neuron-specific enolase (NES) serves as an early predictor of delayed neuropsychiatric sequelae (DNS) in patients with ACMP (5). The diagnostic roles of these serum biochemical indexes, including Lac, CK-MB, LDH, and NSE on DEACMP need to be further investigated.

Diffusion-weighted magnetic resonance imaging (DW-MRI) is a noninvasive medical diagnostic technique that provides highquality three-dimensional or cross-sectional images of tissues or organs (8). The apparent diffusion coefficient (ADC) value determined by DW-MRI has been used for the diagnosis of ACMP complications. For instance, the ADC value measured by early DW-MRI has a predictive value for DNS in patients with ACMP (30). The ADC value of globus pallidus reflects the degree of brain injury in DECAMP patients on a microscopic scale (12). The diagnostic potential of DW-MRI for DEACMP needs to be further investigated.

In this study, we explored the predictive value of serum biochemical markers and ADC on DEACMP. Our research may discover promising biomarkers in the diagnosis of DEACMP.

\section{MATERIAL and METHODS}

\section{Patients}

A total of 79 ACMP patients were admitted at Zibo Municipal Hospital from June 2015 to March 2018. Based on DEACMP onset after 60 days, patients were divided into the DEACMP group and non-DEACMP group. The diagnostic criteria for DEACMP were in line with $\mathrm{WHO}$ recommendations. The clinical data of the ACMP patients were retrospectively analyzed, including their ages, genders, and whether they smoke and/or drink, previous coma cases, duration of poisoning, and the presence of other comorbidities such as hypertension, coronary heart disease, and diabetes. Informed consent was obtained from all patients before collection of samples. This study was approved by the Ethics Committee of Zibo Municipal Hospital in accordance with the Declaration of Helsinki.

\section{Serum Biochemical Indexes}

Blood samples from the vein were taken from the patients within 24 hours of DEACMP onset. White blood cell (WBC) count was measured using an automatic blood cell analyzer (Sysmex, Kobe, Japan). The serum was separated by centrifugation at $2500 \mathrm{~g}$ for $15 \mathrm{~min}$. The serum level of $\mathrm{COHb}$ was measured using a pulse co-oximeter (Masimo Rad-7, Masimo, Irvine, CA, USA), cTnl using a chemiluminescent immunoassay on Access 2 analyzer (Beckman Counter, Brea, California, USA), Lac using a Cobas 6000 analyzer (Roche Diagnostics $\mathrm{GmbH}$, Mannheim, Germany), CK by a colorimetric method using a creatine kinase activity assay kit (Abcam, Cambridge, MA, USA), CKMB isoenzyme using a Beckman CX3 automatic biochemistry analyzer (Hitachi, Tokyo, Japan), LDH using an AU 2700 automatic biochemistry analyzer (Olympus, Tokyo, Japan), NSE using a solid-phase immunoassay with double monoclonal antibodies with an Elecsys 2010 (Roche Diagnostics GmbH, Germany), and CRP using a turbidity method based on latex agglutination on a CRP-Latex (II) X2 reagent Hitachi 7020 analyzer (Hitachi, Tokyo, Japan).

\section{Brain DW-MRI Techniques}

A GE Signa HDxT 1.5T (MRI) (GE, Milwaukee, WI, USA) was used to examine craniocerebral MRI scans within 48 hours after onset of DEACMP. The radiographs were analyzed using an ADW4.4 workstation, and the apparent diffusion coefficient (ADC) values of the globus pallidum, centrum semiovale, and periventricular white matter were determined.

\section{Statistical Analysis}

Statistical analysis was performed with SPSS 23.0 (SPSS Inc., Chicago, IL, USA). Measurement data were presented as means \pm standard deviations (SD) and assessed using a Student's t-test. Enumeration data were presented as numbers $(\mathrm{N})$ and assessed using a $\mathrm{X}^{2}$ test. Logistic regression analysis was performed to identify the independent risk factors for DEACMP. A $p$ value $<0.05$ was considered statistically significant.

\section{RESULTS}

\section{Coma Cases and Duration of Poisoning were Increased in Patients with DEACMP}

The coma cases and duration of poisoning in the DEACMP group were significantly higher than those in the non-DEACMP group $(p<0.01)$. Additionally, there was no significant difference based on sex, age, history of smoking and/or alcoholism, and the presence of comorbidities such as hypertension, coronary heart disease, and diabetes between the two groups $(p>0.05)$ (Table I).

\section{Analysis of Serum Biochemical Values in DEACMP}

Serum levels of COHb, Lac, cTnl, CK, CK-MB, LDH, NSE, $\mathrm{CRP}$, and WBC counts were detected. The results showed 
that serum levels of Lac, CK, CK-MB, LDH, CRP, and NSE were significantly higher in the DEACMP group than those in the non-DEACMP group $(p<0.01)$. However, there was no difference in the serum levels of $\mathrm{COHb}$ and $\mathrm{cTnl}$ as well as WBC counts between the two groups ( $p>0.05$ ) (Table II).

\section{ADC Values of Different Brain Tissues in DEACMP}

The ADC values of the globus pallidum, centrum semiovale, and periventricular white matter were determined by DW-MRI.

The results found the ADC value of globus pallidum in the
DEACMP group to be lower than that in the non-DEACMP group $(p<0.01)$. However, there was no statistical difference between these two groups in terms of ADC values of the centrum semiovale and periventricular white matter $(p>0.05)$ (Table III).

\section{Logistic Regression Analysis of Risk Factors for DEACMP}

Logistic regression analysis showed that there was a significant correlation between DEACMP and the duration of poisoning $(p<0.05)$, coma degree $(p<0.05)$, CK $(p<0.001)$, LDH $(p<0.05)$,

Table I: Baseline Data for DEACMP and no-DEACMP Groups

\begin{tabular}{lccc}
\hline Items & DEACMP group $(\mathbf{n}=\mathbf{3 4})$ & no-DECAMP group $(\mathbf{n}=\mathbf{4 5})$ & $\mathbf{p}$ \\
\hline Gender (male/female) & $21 / 13$ & $26 / 19$ & 0.721 \\
\hline Age (years old) & $57.34 \pm 17.34$ & $52.43 \pm 15.42$ & 0.195 \\
\hline Smoke & 14 & 24 & 0.284 \\
\hline Drink alcohol & 16 & 24 & 0.581 \\
\hline Hypertension & 15 & 25 & 0.314 \\
\hline Coronary heart disease & 12 & 20 & 0.412 \\
\hline Diabetes & 13 & 21 & 0.454 \\
\hline Coma & $27^{* *}$ & 18 & $<0.001$ \\
\hline Duration of poisoning (hour) & $22.54 \pm 10.25^{* *}$ & $13.41 \pm 16.78$ & 0.006 \\
\hline
\end{tabular}

${ }^{* *} p<0.01$ vs. no-DECAMP group.

Table II: Biochemical Values Between DEACMP and no-DEACMP Groups

\begin{tabular}{lcccc}
\hline Biochemical values & DEACMP group & No-DEACMP group & $\mathbf{p}$ & F value \\
\hline $\mathrm{COHb}(\%)$ & $21.24 \pm 18.11$ & $24.01 \pm 13.89$ & 0.444 & 0.588 \\
\hline $\mathrm{CTnl}(\mathrm{mg} / \mathrm{L})$ & $1.62 \pm 3.33$ & $0.48 \pm 1.76$ & 0.053 & 0.279 \\
\hline $\mathrm{WBC}(/ \mu \mathrm{L})$ & $14287.23 \pm 3786.46$ & $13784.24 \pm 8946.13$ & 0.759 & 5.582 \\
\hline $\mathrm{Lac}(\mathrm{U} / \mathrm{L})$ & $6.11 \pm 2.54^{*}$ & $5.08 \pm 1.63$ & 0.032 & 0.412 \\
\hline $\mathrm{CK}(\mathrm{IU} / \mathrm{L})$ & $4231.18 \pm 5346.17^{* *}$ & $721.34 \pm 2332.14$ & 0.0002 & 0.190 \\
\hline $\mathrm{CK}-\mathrm{MB}(\mathrm{IU} / \mathrm{L})$ & $51.32 \pm 33.12^{*}$ & $36.72 \pm 23.24$ & 0.024 & 0.492 \\
\hline $\mathrm{LDH}(\mathrm{U} / \mathrm{L})$ & $384.67 \pm 276.45^{* *}$ & $231.22 \pm 108.64$ & 0.001 & 0.154 \\
\hline $\mathrm{CRP}(\mathrm{mg} / \mathrm{L})$ & $30.34 \pm 36.28^{* *}$ & $4.12 \pm 2.57$ & $<0.0001$ & 0.005 \\
\hline $\mathrm{NSE}(\mathrm{U} / \mathrm{mL})$ & $18.73 \pm 5.46^{* *}$ & $11.98 \pm 4.16$ & $<0.0001$ & 0.580 \\
\hline
\end{tabular}

${ }^{*} p<0.05,{ }^{* *} p<0.01$ vs. no-DECAMP group.

Table III: ADC Values of Different Brain Tissues Between DEACMP and no-DEACMP Groups

\begin{tabular}{lcccc}
\hline Position $\left(\times \mathbf{1 0} \mathbf{3 m}^{-3} \mathbf{m}\right)$ & DEACMP group & No-DEACMP group & $\mathbf{p}$ & F value \\
\hline Globus pallidum & $0.52 \pm 0.07^{* *}$ & $0.76 \pm 0.04$ & $<0.0001$ & 0.327 \\
\hline Semi-soft circle & $0.67 \pm 0.07$ & $0.71 \pm 0.16$ & 0.177 & 5.224 \\
\hline Periventricular white matter & $0.70 \pm 0.06$ & $0.72 \pm 0.05$ & 0.110 & 0.694 \\
\hline
\end{tabular}

${ }^{* *} \mathrm{p}<0.01$ vs. no-DECAMP group. 
Sun R. et al: Serum Biochemical Markers and ADC in DEACMP

Table IV: Logistic Regression Analysis of DEACMP Related Risk Factors

\begin{tabular}{lccccc}
\hline Items & $\boldsymbol{\beta}$ & $\mathbf{S E}$ & Wald & P value & Exp ( $\boldsymbol{\beta})$ \\
\hline Coma degree & -0.096 & 0.057 & 0.624 & 0.008 & 0.872 \\
\hline Duration of poisoning & 0.014 & 0.008 & 5.624 & 0.019 & 1.048 \\
\hline Lac & -0.412 & 0.204 & 3.683 & 0.056 & 0.672 \\
\hline NSE & -0.654 & 0.326 & 5.118 & 0.027 & 0.514 \\
\hline CK & 0.064 & 0.026 & 12.859 & $<0.0001$ & 1.069 \\
\hline CK-MB & 0.285 & 0.231 & 1.695 & 0.181 & 1.302 \\
\hline CRP & -0.145 & 0.057 & 9.215 & 0.002 & 0.826 \\
\hline LDH & 1.313 & 0.564 & 4.625 & 0.026 & 3.326 \\
\hline ADC value of Globus pallidum & 0.354 & 0.386 & 0.849 & 0.361 & 1.462 \\
\hline
\end{tabular}

NES $(p<0.05)$, and CRP $(p<0.01)$ (Table IV). The above indices were possible independent risk factors for DEACMP with statistically significant differences.

\section{DISCUSSION}

DEACMP is a CO poisoning-induced disease with poor prognosis (15). CO poisoning causes both tissue hypoxia and direct cellular changes causing inflammatory or immunological injury $(36,41)$. Prolonged exposure to $\mathrm{CO}$ leads to the development of neurologic sequelae, especially delayed encephalopathy $(25,29)$. Song et al. have presented that approximately $10 \%$ to $30 \%$ patients who had $\mathrm{CO}$ poisoning develop DEACMP (32). Du et al. have shown that patients with DEACMP have a longer exposure to $\mathrm{CO}$ compared with those without DEACMP (9). In this study, the duration of exposure to $\mathrm{CO}$ in the DEACMP group was significantly higher than that in the non-DEACMP group. Our results are consistent with previous studies and illustrate that CO poisoning can cause DEACMP. Coma is one of the most obvious clinical manifestations of $\mathrm{CO}$ poisoning (27). Prolonged duration of coma after ACMP is also a risk factor for DEACMP $(14,34)$. Pan et al. have shown that prolonged duration of coma for 11 hours may result in a markedly increased incidence rate of DEACMP (14). Taki and Nakajima have found that ACMP patients with coma have a higher incidence of DEACMP than ACMP patients without coma (34). In this study, the number of coma cases in the DEACMP group was also significantly higher than those in the non-DEACMP group, indicating that coma is closely related to the onset of DEACMP. Furthermore, upon logistic regression analysis, the duration of poisoning and coma degree were found to be independent risk factors for DEACMP. Therefore, special attention should be paid to the occurrence of DEACMP in ACMP patients with long duration of poisoning and high degree of coma.

Many serum biochemical markers which may be able to determine the degree of DEACMP have been studies, which include Lac (22), CK, CK-MB (20,31), and LDH (18). Lac is a product of anaerobic glycolysis under low oxygen supply that can act as an indicator for early CO poisoning (7). Moon et al. have indicated that Lac is associated with short-term outcomes in patients with ACMP, as patients with high initial Lac level exhibit a high incidence of DEACMP (22). CK and CK-MB are intracellular enzymes predominantly located in skeletal muscle, myocardium, and the brain (3). High CK and CK-MB levels can be induced by CO-induced damage in multiple organs in patients with ACMP. Thus, serum CK and CK-MB levels can be used as predictors for ACMPassociated diseases $(19,38)$. LDH is an important enzyme of the anaerobic metabolic pathway (10). Winburn et al. have found that exposure of human erythrocytes to exogenous CO moderately inhibited LDH activity (42). Kudo et al. have reported the levels of CK, CK-MB and LDH to be markedly increased in DEACMP patients (18). In this study, the serum levels of Lac, CK, CK-MB and LDH in the DEACMP group were significantly higher than those in the non-DEACMP group. Our results indicate that these serum biochemical markers are closely associated with the occurrence of DEACMP, which are consistent with previous studies. Furthermore, logistic regression analysis found increased serum levels of $\mathrm{CK}$ and $\mathrm{LDH}$ to be independent risk factors for DEACMP. These results indicate that $\mathrm{CK}$ and $\mathrm{LDH}$ may act as promising predictive factors of DEACMP in clinical practice.

CRP is a common acute phase protein that can be elevated by hypoxia, trauma and other stress states in human organisms $(23,43)$. Sawiniec et al. have proved CRP levels to have diagnostic value for ACMP, with CRP levels in ACMP patients found to increase by over 33\% (28). Song et al. have shown that serum CRP level is useful for the early diagnosis and prognostic assessment of DEACMP (32). NSE is a marker of the condition of the central nervous system, with elevations in its levels closely related to the degree of brain damage $(4,44)$. Yildirim et al. have demonstrated that serum NSE level is an indicator for brain injury in CO poisoning patients (45). Cha et al. have also reported that serum NES acts as an early predictor of DNS in patients with ACMP (5). In this study, serum levels of CRP and NSE in the DEACMP group were significantly higher than those in the non-DEACMP group. In addition, logistic regression analysis showed that increased serum levels of CRP and NES were independent risk factors 
for DEACMP. Our results illustrate that CK and LDH may be promising predictive factors of DEACMP.

MRI is an important diagnostic tool to identify ACMP patients who have developed chronic neurological symptoms (16). It can reveal neurological lesions resulting from DEACMP that are mainly located in the globus pallidus, subcortical white matter, and basal ganglia (40). The globus pallidus is a vulnerable structure in patients who had $\mathrm{CO}$ poisoning, including ACMP (6). Ischemic lesions in patients with ACMP are easily reflected in globus pallidus (24). Bleecker have reported that the globus pallidus was the most commonly affected area of the brain following CO poisoning (2). Hopkins et al. have observed bilateral lesions in the globus pallidus after $C O$ exposure (13). ADC values measured by DWI is an effective indicator of brain dysfunction $(17,37)$. Bang and Kim have reported the ADC values of the globus pallidus in DNS patients to be lower than that in non-DNS patients (1). Moon et al. have shown the ADC value of globus pallidus to be decreased in patients with ACMP (21). In this study, the $A D C$ value of globus pallidus in the non-DEACMP group was significantly higher than that in the DEACMP group. This result is consistent with previous studies and indicates that the ADC value of globus pallidus can reflect the onset of DEACMP to some extent. However, logistic regression analysis showed that a decreased ADC value of the globus pallidus was not an independent risk factor for DEACMP, indicating that the predictive value of the ADC value of the globus pallidus for DEACMP is limited. Further studies on the predictive value of DW-MRI on DEACMP therefore need to be further investigated.

\section{CONCLUSION}

In conclusion, the serum levels of Lac, CK, CK-MB, LDH, CRP, and NSE in ACMP patients were positively related to DEACMP, while ADC value of the globus pallidus in ACMP patients was negatively related to DEACMP. Additionally, the duration of poisoning, coma degree, CK, LDH, NES, and CRP were independent risk factors for DEACMP.

\section{REFERENCES}

1 Bang SJ, Kim GT: Can predict late encephalopathy by diffusion tensor imaging in acute phase of carbon monoxide poisoning? J Korean Soc Emerg Med 26:179-188, 2015

2 Bleecker ML: Carbon monoxide intoxication. Handb Clin Neurol 131: 191-203, 2015

3 Bojinca M, Bojinca VC, Balanescu AR, Balanescu SM: Macro creatine kinase (macro CK) in clinical practice. Revista De Chimie Bucharest Original Edition 69: 2107-2109, 2018

4 Cakir Z, Aslan S, Umudum Z, Acemoglu H, Akoz A, Turkyilmaz S, Ozturk N: S-100 beta and neuron-specific enolase levels in carbon monoxide-related brain injury. Am J Emerg Med 28: 61-67, 2010

5 Cha Y, Kim H, Do H, Kim H, Kim O, Cha K, Lee K, Hwang $\mathrm{S}$ : Serum neuron-specific enolase as an early predictor of delayed neuropsychiatric sequelae in patients with acute carbon monoxide poisoning. Hum Exp Toxicol 37:240-246, 2018
6 Chen NC, Huang CW, Lui CC, Lee CC, Chang WN, Huang $\mathrm{SH}$, Chen C, Chang CC: Diffusion-weighted imaging improves prediction in cognitive outcome and clinical phases in patients with carbon monoxide intoxication. Neuroradiology 55:107115, 2013

7 Dogan N: The role of lactic acid in carbon monoxide poisoning and the difficulty of defining the worse outcome. Hum Exp Toxicol 35:795,796, 2016

8 Dolgushin M, Kornienko V, Pronin I: Magnetic Resonance Imaging (MRI). In: Neuroimaging Techniques in the Diagnosis of Brain Cancer Metastases, Springer, 2018:51-83

9 Du X, Gu H, Hao F, Gao L, Wang J, Sun C, Zhang H, Ma P, Zhang $\mathrm{X}, \mathrm{Hu}$ W: Utility of brain CT for predicting delayed encephalopathy after acute carbon monoxide poisoning. Exp Ther Med 17:2682-2688, 2019

10 Farhana A, Lappin SL: Biochemistry, Lactate Dehydrogenase (LDH). In: StatPearls [Internet]. Treasure Island (FL): StatPearls Publishing, 2020

11. Goldstein M: Carbon monoxide poisoning. J Emerg Nurs 34(6): 538-542, 2008

12. Guo J, Meng J, Han T: MRI-based comparison of brain damage between acute carbon monoxide poisoning and delayed encephalopathy after acute carbon monoxide poisoning. Zhonghua Lao Dong Wei Sheng Zhi Ye Bing Za Zhi 32:533-536, 2014

13. Hopkins RO, Fearing MA, Weaver LK, Foley JF: Basal ganglia lesions following carbon monoxide poisoning. Brain Inj 20:273-281, 2006

14. Hou X, Ma L, Wu L, Zhang Y, Ge H, Li Z, Gao Y, Zhou Y, Gao C: Diffusion tensor imaging for predicting the clinical outcome of delayed encephalopathy of acute carbon monoxide poisoning. Eur Neurol 69:275-280, 2013

15. Hu H, Pan X, Wan Y, Zhang Q, Liang W: Factors affecting the prognosis of patients with delayed encephalopathy after acute carbon monoxide poisoning. Am J Emerg Med 29:261264, 2011

16. Jeon SB, Sohn CH, Seo DW, Oh BJ, Lim KS, Kang DW, Kim $W Y$ : Acute brain lesions on magnetic resonance imaging and delayed neurological sequelae in carbon monoxide poisoning. JAMA Neurol 75:436-443, 2018

17. Jissendi Tchofo P, Christophe C, David P, Metens T, Soto Ares G, Baleriaux D: Apparent diffusion coefficient (ADC) and magnetization transfer ratio (MTR) in pediatric hypoxicischemic brain injury. J Neuroradiol 32:10-19, 2005

18. Kudo K, Otsuka K, Yagi J, Sanjo K, Koizumi N, Koeda A, Umetsu M, Yoshioka Y, Mizugai A, Mita T: Predictors for delayed encephalopathy following acute carbon monoxide poisoning. BMC Emerg Med 14:3, 2014

19. Lee H, Kang H, Ko BS, Oh J, Lim TH, Cho Y: Initial creatine kinase level as predictor for delayed neuropsychiatric sequelae associated with acute carbon monoxide poisoning. Am J Emerg Med, 2020 (Online ahead of print)

20. Lee I, Jung Y, Kim G, Min Y, Park E, Lee JH, Choi S: What is the meaning of cardiac toxicities in the clinical course of acute carbon monoxide poisoning. J Emerg Med 43:931, 2012

21. Moon J, Chun B, Baek B, Hong Y: Initial diffusion-weighted $\mathrm{MRI}$ and long-term neurologic outcomes in charcoal-burning carbon monoxide poisoning. Clinical Toxicology 56:161-169, 2018 
22. Moon JM, Shin MH, Chun BJ: The value of initial lactate in patients with carbon monoxide intoxication in the emergency department. Hum Exp Toxicol 30:836-843, 2011

23. Neumaier M, Metak G, Scherer MA: C-reactive protein as a parameter of surgical trauma: CRP response after different types of surgery in 349 hip fractures. Acta Orthop 77(5):788790, 2006

24. O'donnell P, Buxton P, Pitkin A, Jarvis L: The magnetic resonance imaging appearances of the brain in acute carbon monoxide poisoning. Clin Radiol 55(4):273-280, 2000

25. Pepe G, Castelli M, Nazerian P, Vanni S, Del Panta M, Gambassi F, Botti P, Missanelli A, Grifoni S: Delayed neuropsychological sequelae after carbon monoxide poisoning: Predictive risk factors in the Emergency Department. A retrospective study. Scand J Trauma Resusc Emerg Med 19:16, 2011

26. Raphaël JC: Acute carbon monoxide poisoning. La Revue Du Praticien 58:849-854, 2008

27. Rose JJ, Wang L, Xu Q, Mctiernan CF, Shiva S, Tejero J, Gladwin MT: Carbon monoxide poisoning: Pathogenesis, management, and future directions of therapy. Am J Respir Crit Care Med 195(5):596-606, 2017

28. Sawiniec J, Gnyp L, Lewandowska-Stanek H: C-reactive protein as a useful prognostic marker in acute poisoning. Przegl Lek 61:356-358, 2004

29. Sekiya K, Nishihara T, Abe N, Konishi A, Nandate H, Hamada T, Ikemune K, Takasaki Y, Tanaka J, Asano M, Yorozuya T: Carbon monoxide poisoning-induced delayed encephalopathy accompanies decreased microglial cell numbers: Distinctive pathophysiological features from hypoxemia-induced brain damage. Brain Res 1710:22-32, 2019

30. Sener RN: Acute carbon monoxide poisoning: Diffusion MR imaging findings. AJNR Am J Neuroradiol 24:1475-1477, 2003

31. Song I, Chung S: Chorea as the first neurological symptom of delayed encephalopathy after carbon monoxide intoxication. Intern Med 49(11):1037-1039, 2010

32. Song SS, Li Y, Niu L, Liu S, Duan CF, Sui QL, Zhao JP, Ren YD, Liu XJ: Delayed encephalopathy after acute carbon monoxide poisoning with dynamic progression of radiologic and clinical manifestations. Neuropsychiatry 8(6):1893-1898, 2018

33. Sung PS, Yu CY, Lin TS: Asymmetrical delayed encephalopathy after acute $\mathrm{CO}$ intoxication: A case report. NeuroToxicology 31(1):161-163, 2010

34. Taki K, Nakajima S: Potential application of hyperbaric oxygen therapy (HBOT) to carbon monoxide poisoning: Analysis of intermittent CO poisoning cases. Jpn J Reanimatology 26:107-112, 2007

35. Terajima K, Igarashi $H$, Hirose $M$, Matsuzawa $H$, Nishizawa M, Nakada T: Serial assessments of delayed encephalopathy after carbon monoxide poisoning using magnetic resonance spectroscopy and diffusion tensor imaging on 3.0T system. Eur Neurol 59:55-61, 2008
36. Thom SR, Bhopale VM, Fisher D: Hyperbaric oxygen reduces delayed immune-mediated neuropathology in experimental carbon monoxide toxicity. Toxicol Appl Pharmacol 213:152159, 2006

37. Villanueva-Meyer JE, Mabray MC, Cha S: Current clinical brain tumor imaging. Neurosurgery 81:397-415, 2017

38. Wang P, Li W, Yang JL, Wang Y, Liu Q, Wang WZ, Qi HN, Li J: Effect of ulinastatin on myocardial injury in patients with acute severe carbon monoxide poisoning. Zhonghua Lao Dong Wei Sheng Zhi Ye Bing Za Zhi 36(2):154-156, 2018

39. Wang WZ, Ma GY, Zhao LJ, Li J, Wang P, Li YQ, Xiao QM, Liu YJ: The relationship between lactate clearance rate and delayed encephalopathy after acute carbon monoxide poisoning. Zhonghua Wei Zhong Bing Ji Jiu Yi Xue 25:622626, 2013

40. Wang X, Li Z, Berglass J, He W, Zhao J, Zhang M, Gao C, Zhang $\mathrm{C}$, Zhang $\mathrm{H}$, Yi X: MRI and clinical manifestations of delayed encephalopathy after carbon monoxide poisoning. Pak J Pharm Sci 29:2317-2320, 2016

41. Weaver LK: Clinical practice. Carbon monoxide poisoning. $\mathrm{N}$ Engl J Med 360:1217-1225, 2009

42. Winburn IC, Gunatunga K, Mckernan RD, Walker RJ, Sammut IA, Harrison JC: Cell damage following carbon monoxide releasing molecule exposure: Implications for therapeutic applications. Basic Clin Pharmacol Toxicol 111:31-41, 2012

43. Yang J, Wang J, Zhu S, Chen X, Wu H, Yang D, Zhang J: C-reactive protein augments hypoxia-induced apoptosis through mitochondrion-dependent pathway in cardiac myocytes. Mol Cell Biochem 310:215-226, 2008

44. Yardan T, Cevik Y, Donderici O, Kavalci C, Yilmaz FM, Yilmaz G, Vural K, Yuzbasioglu Y, Gunaydin YK, Sezer AA: Elevated serum S100B protein and neuron-specific enolase levels in carbon monoxide poisoning. Am J Emerg Med 27:838-842, 2009

45. Yildirim AO, Eroglu M, Kaldirim U, Eyi YE, Simsek K, Durusu M, Yamanel L, Arziman I, Tuncer SK, Toygar M, Balkan A, Cayci T, Demirbas S, Oter S, Bilgi C: Serum neuron-specific enolase and S-100beta levels as prognostic follow-up markers for oxygen administered carbon monoxide intoxication cases. Indian J Biochem Biophys 52:29-33, 2015

46. Zhang JG, Zhang HR, Shi XY, Zhao Y, Su Y, Li XZ, Su XC, Miao $Z Y$ : Changes of myocardial enzymes in patients with acute carbon monoxide poisoning. Zhonghua Lao Dong Wei Sheng Zhi Ye Bing Za Zhi 21:51-53, 2003

47. Zhou W, Feng X, Ren C, Jiang X, Liu W, Huang W, Liu Z, Li Z, Zeng L, Wang L: Over-expression of BCAT1, a c-Myc target gene, induces cell proliferation, migration and invasion in nasopharyngeal carcinoma. Mol Cancer 12:53, 2013 